\title{
Incidence, clinical outcome, and risk stratification of ventilator-associated pneumonia-a prospective cohort study
}

\author{
Panwar Rakshit, Vidya S. Nagar, Alaka K. Deshpande
}

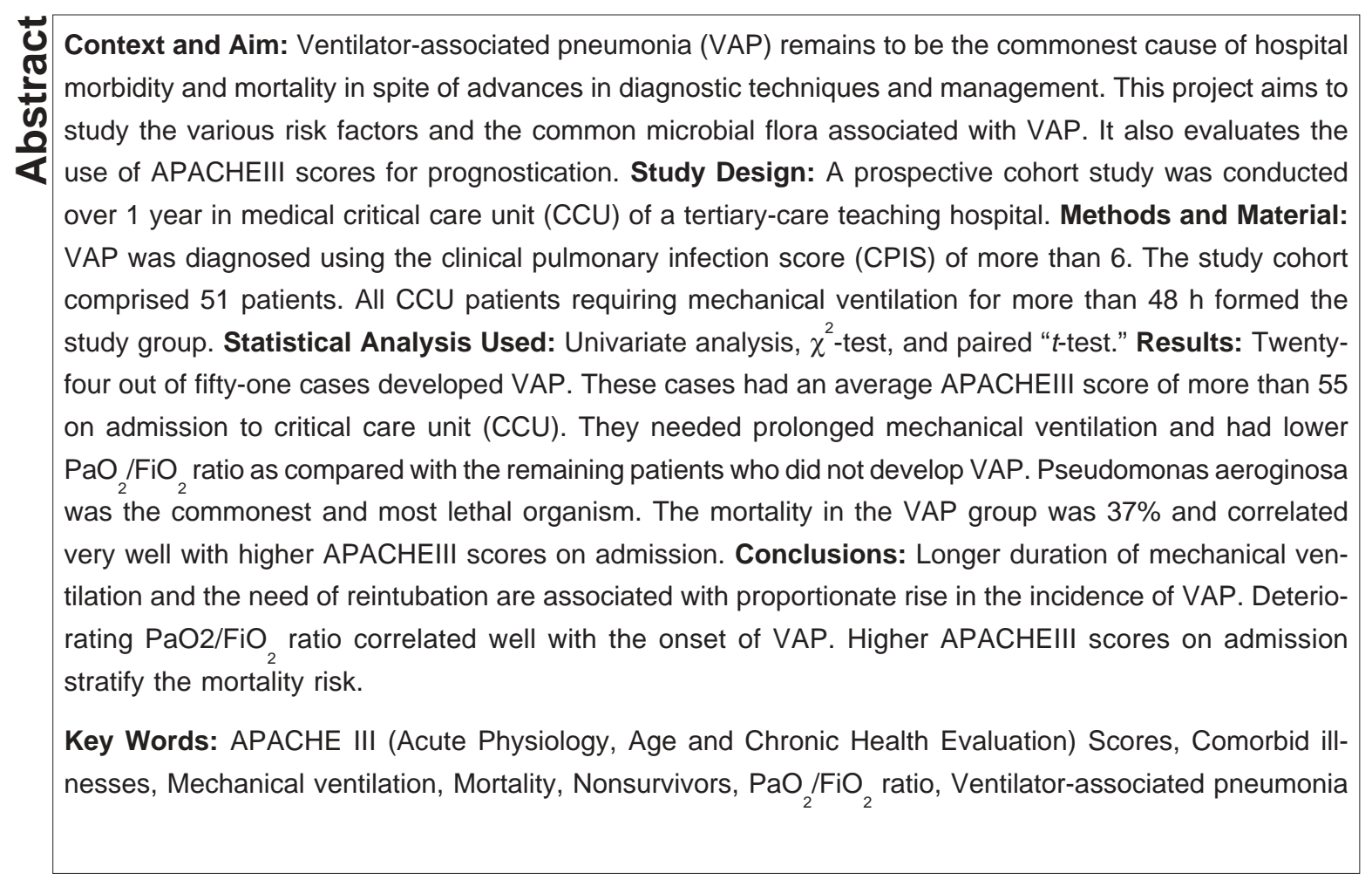

\section{Introduction}

Ventilator-associated pneumonia (VAP) refers to bacterial pneumonias developing in patients who have been receiving mechanical ventilation (MV) for at least $48 \mathrm{~h} \cdot{ }^{[1]}$

VAP is the commonest complication associated with

\section{From:}

Department of Medicine, Grant Medical College, Sir J. J. Group of Government Hospitals, Mumbai, India

\section{Correspondence:}

Panwar Rakshit, A-1203, Eternia, Hiranandani Gardens, Powai, Mumbai 400076, India. E-mail: rakshitpanwar@hotmail.com
MV reported at the rate of $1-3 \%$ per day of MV. ${ }^{[2]}$ Prevalence ranges from $10 \%$ to $65 \%$ in tertiary-care hospitals with pari passu rise in case fatality rates of more than $20 \%$ according to reported studies. ${ }^{[3]}$ Incriminating pathogens vary among hospitals. ${ }^{[4]}$ Therefore, incidence of VAP and the associated local microbial flora needs to be studied in each setting so as to guide more effective and rational utilization of antimicrobial agents. ${ }^{[4]}$

The precise diagnosis of pneumonia is often difficult in critically ill patients. Purulent sputum may follow intuba- 
tion or leakage of oropharyngeal secretions around the artificial airway. Furthermore, chest roentgenographic changes consistent with pneumonia may be caused by pulmonary edema, pulmonary infarction, atelectasis, or acute respiratory distress syndrome..$^{[5]}$ Lack of an unequivocal gold standard complicates the issue of deciding the best diagnostic technique for suspected VAP. Although the sensitivity of a clinical diagnosis of VAP is high, the specificity is low. But VAP is one entity in which a subjective clinical impression appears to be more accurate than a set of objective criteria. ${ }^{[6]}$

APACHEIII score, which is a further refinement of APACHE system, may help in stratifying hospital mortality risk, which may impact on the scope and intensity of treatment.

This study was designed to determine the incidence of VAP, the associated risk factors, the causative microbial flora, and the role of APACHEIII in prognostication of patients at the time of admission to CCU in a tertiarycare centre.

\section{Materials and Methods}

The study spanned a period of 1 year. All cases admitted in $\mathrm{CCU}$ in past 1 year requiring $\mathrm{MV}$ for longer than $48 \mathrm{~h}$ formed the study cohort.

Patients who were already on MV before admission to $\mathrm{CCU}$ or those who died within $48 \mathrm{~h}$ of starting MV were excluded.

VAP was considered as a subjective clinical impression. It was further substantiated using clinical pulmonary infection score (CPIS). CPIS of greater than 6 was used as diagnostic criteria for VAP. ${ }^{[7]}$ Pugin et al..[] developed a CPIS score based on giving 0-2 points each for fever, leukocyte count, oxygenation, quantity, and purulence of tracheal secretions, type of radiographic abnormality, and results of sputum culture and Gram stain.

The baseline evaluation of all the cases in this study included the patient-related factors such as age, concomitant diseases, immunosuppression, indication of $\mathrm{MV}$, the ratio of $\mathrm{PaO} 2$ to $\mathrm{FiO} 2$ prior to onset of $\mathrm{VAP}$, and severity of illness based on APACHEIII scoring system.
On admission to CCU, the entire cohort was allotted scores for their age, chronic health status, physiologic parameters, acid-base status, and neurological status, based on APACHEIII scoring system. Total APACHEIII score was deduced by adding all these scores. ${ }^{[8]}$

The risk factors included reintubation, use of chemical paralysis, need for dialysis, duration of MV, and duration of CCU stay.

\section{Statistical Analysis}

The study cohort was classified in four groups: VAP, non-VAP, survivors, and nonsurvivors. After evaluating all the above-mentioned factors, the data were subjected to the univariate analysis using the chi-square test. The level of significance was set at $P<0.05$.

\section{Results}

The study cohort comprised 51 patients with 29 (56.9\%) males and $22(43.1 \%)$ females. The mean age of patients in this cohort was 34 years; 24 out of 51 patients (47\%) developed VAP during CCU stay. The incidence rate of VAP in our study was 26 per 1000 ventilator days.

Table 1 shows the clinical spectrum of our cases that includes 21 patients with various neurological disorders, 6 cases of tropical diseases, 12 cases of poisonings, 12 cases of metabolic disorder, 3 cases of sepsis, and others. Some of our patients had multiple disorders. The incidence of VAP was greater either in patients with diseases necessitating prolonged MV (e.g., Guillain-Barre syndrome, tetanus, organophosphorus poisoning, etc.), or in patients with those diseases that predispose to pulmonary infection (such as sepsis, tropical diseases, or immunosuppression) (Table 1).

It is also significant to note that the incidence of VAP was considerably low in patients with diseases which presumably, had unaffected lungs before admission to CCU, for example, meningoencephalitis (12\%), hydrocephalus $(0 \%)$, snake bite $(0 \%)$, etc. (Table 1$)$.

Table 2 shows the distribution of onset of VAP for the study cohort. The total $(n)$ number of patients on MV who were being followed during each time period is given along with the percentage of those people who did not develop VAP. Also given is the number of cases with onset of VAP during each time period. This table clearly 
Table 1: Clinical spectrum

\begin{tabular}{|c|c|c|c|c|}
\hline \multicolumn{2}{|c|}{ Diseases } & $\begin{array}{l}n \text { (no. of } \\
\text { patients) }\end{array}$ & VAP & Non-VAP \\
\hline \multicolumn{5}{|c|}{ CNS disorders } \\
\hline \multicolumn{2}{|c|}{ Meningoencephalitis } & 8 & $1(12 \%)$ & 7 \\
\hline \multicolumn{2}{|c|}{ GBS/AIDP } & 8 & $4(50 \%)$ & 4 \\
\hline \multicolumn{2}{|c|}{ Myasthenia gravis } & 1 & 1 & NA \\
\hline \multicolumn{2}{|c|}{ Hydrocephalus/SAH } & 4 & NA $(0 \%)$ & 4 \\
\hline \multicolumn{5}{|c|}{ Tropical diseases } \\
\hline \multicolumn{2}{|c|}{ Complicated malaria } & 2 & $1(50 \%)$ & 1 \\
\hline \multicolumn{2}{|c|}{ Leptospirosis } & 1 & 1 & - \\
\hline \multicolumn{2}{|c|}{ Tetanus } & 3 & $2(67 \%)$ & 1 \\
\hline \multicolumn{5}{|c|}{ Poisoning } \\
\hline \multicolumn{2}{|c|}{ OP poisoning } & 11 & $6(55 \%)$ & 5 \\
\hline \multicolumn{2}{|c|}{ Snake bite } & 1 & $-(0 \%)$ & 1 \\
\hline \multicolumn{5}{|c|}{ Metabolic } \\
\hline \multicolumn{2}{|c|}{ ARF/CRF/RTA } & 10 & $5(50 \%)$ & 5 \\
\hline \multicolumn{2}{|c|}{ DM/DKA } & 2 & $1(50 \%)$ & 1 \\
\hline \multicolumn{5}{|c|}{ Miscellaneous } \\
\hline \multicolumn{2}{|c|}{ Shock/sepsis } & 3 & $2(67 \%)$ & 1 \\
\hline \multicolumn{2}{|c|}{ Pancreatitis } & 2 & 1 & 1 \\
\hline \multicolumn{2}{|c|}{ Pulmonary fibrosis } & 1 & 1 & 0 \\
\hline \multicolumn{5}{|c|}{$\begin{array}{l}\text { Total }(n) \text { is more than } 51 \text { as some patients had multiple disorders. Percent- } \\
\text { age in parentheses denotes percentage of patients with the specified } \\
\text { disease, who developed VAP. CNS, central nervous system; GBS, Guillain- } \\
\text { Barre syndrome; AIDP, acute inflammatory demyelinating polyneuropathy; } \\
\text { SAH, subarachnoid hemorrhage; OP, organophosphorous; ARF, acute renal } \\
\text { failure; CRF, chronic renal failure; RTA, renal tubular acidosis; DM, diabetes } \\
\text { mellitus; DKA, diabetic ketoacidosis. }\end{array}$} \\
\hline \multicolumn{5}{|c|}{$\begin{array}{l}\text { Table 2: Distribution of onset of VAP for the cohort and } \\
\text { cumulative risk of VAP with duration of MV }\end{array}$} \\
\hline $\begin{array}{l}\text { Days of } \\
\text { MV }\end{array}$ & Total $(n)$ & $\begin{array}{l}\text { No. of patients } \\
\text { with onset of VAP }\end{array}$ & $\begin{array}{l}\text { No. free } \\
\text { of VAP }\end{array}$ & $\begin{array}{c}\text { Free of VAP } \\
(\%)\end{array}$ \\
\hline $2-4$ & 51 & 8 & 43 & 84.3 \\
\hline $5-10$ & 47 & 7 & 35 & 74.5 \\
\hline $11-15$ & 33 & 3 & 20 & 60.6 \\
\hline $16-20$ & 22 & 3 & 9 & 40.9 \\
\hline $21-25$ & 14 & 2 & 5 & 35.7 \\
\hline$>26^{a}$ & 10 & 1 & 5 & 50 \\
\hline
\end{tabular}

a The time period (26-45) is much longer compared with others.

demonstrates the decline in percentage of patients free of VAP as the duration of MV increases. Patients who developed VAP within $96 \mathrm{~h}$ of MV were categorized as having "early-onset VAP" and those who developed after $96 \mathrm{~h}$ were classified as "late-onset VAP." Eight patients developed early-onset VAP and 16 (31\%) patients developed late-onset VAP. The incidence of VAP increased with the duration of MV. The mean duration of $\mathrm{MV}$ in cases without VAP was 13.5 days as against 23.4 days in patients with VAP $(P<0.05)$. In addition, it may be noted that the mean duration of MV in patients with late-onset VAP was 29.25 days $(P<0.005)$. Thus, it emphasizes a direct correlation between the duration of $\mathrm{MV}$ and development of VAP. The average length of stay in CCU for patients who developed VAP was 28 days $(\sigma=18)$, whereas the average for non-VAP patients was appreciably low at 19 days $(\sigma=13)(P<0.05)$.
Table 3 shows correlation between patient related factors and Incidence of VAP. It also highlights the association between the severity of illness, assessed by APACHEIII scores on admission, and VAP. Patients with VAP had an average APACHEIII score of 55.1, compared with non-VAP group, whose score was $41.75(P<$ $0.05)$ The comorbid conditions played a major role in CCU complications. Patients who developed VAP were more likely to be suffering from conditions causing immunosuppression, such as chronic renal failure, diabetes mellitus, and steroid therapy. These patients were presumably harbouring infections prior to admission.

$\mathrm{PaO}_{2} / \mathrm{FiO}_{2}$ ratio was compared in both the groups (VAP and non-VAP) and was significantly lower in VAP group $(P<0.001) . \mathrm{PaO}_{2} / \mathrm{FiO}_{2}$ ratio was assessed daily during the course of ventilatory support and it was observed that the ratio dropped at least 12-24 h prior to onset of clinicoradiologic picture suggestive of VAP. Patients who were diagnosed ARDS on basis of $\mathrm{PaO}_{2} / \mathrm{FiO}_{2}$ ratio being less than 200, were excluded from this comparison of $\mathrm{PaO}_{2} / \mathrm{FiO}_{2}$ ratio. Thus a decline in $\mathrm{PaO}_{2} / \mathrm{FiO}_{2}$ ratio was found to be an early indicator of the onset of VAP.

Table 4 relates the health-care factors to the VAP. Out of 24 cases, who were reintubated, 19 developed VAP $(P<0.001)$, whereas out of 11 patients, who had early and planned tracheostomy, only one developed VAP $(P<0.005)$.

Table 5 reveals the incriminating microbial flora. Gramnegative organisms were isolated from 26 out of 32 cultures. Most common offending organism isolated in cases with Early-onset VAP is Pseudomonas aeroginosa

\section{Table 3: Patient-related factors}

Comorbid factors $(n=13)$

Immunosuppression/infection $(n=7)$

$\mathrm{PaO}_{2} / \mathrm{FiO}_{2}{ }^{\text {a }}$ ratio

APACHEIll score

\begin{tabular}{ccc} 
Non-VAP & VAP & $\mathbf{P}$ \\
6 & 7 & - \\
2 & 5 & 0.24 \\
294.9 & 211.3 & $<0.001$ \\
41.75 & 55.1 & 0.05 \\
\hline
\end{tabular}

Data expressed as no. unless specified. " $n$ " denotes number of patients.

${ }^{a}$ Few patients were excluded as they were suspected/proven cases of ARDS

\begin{tabular}{lccc}
\hline Table 4: Factors related to course of care \\
\hline & Non-VAP & VAP & $\boldsymbol{P}$ \\
Dialysis $(n=11)$ & 5 & 6 & - \\
Reintubation $(n=24)$ & 5 & 19 & $<0.001$ \\
Tracheostomy $(n=11)$ & 10 & 1 & $<0.005$ \\
Chemical paralysis $(n=8)$ & 3 & 5 & - \\
\hline
\end{tabular}


Table 5: Causative organisms and VAP

\begin{tabular}{lccc}
\hline Organisms & $\begin{array}{c}\text { Early-onset } \\
\text { VAP }\end{array}$ & $\begin{array}{c}\text { Late-onset } \\
\text { VAP }\end{array}$ & Total \\
P. aeroginosa & 6 & 5 & 11 \\
Klebsiella pneumoniae & 2 & 5 & 7 \\
E. coli and Proteus sp. & 1 & 5 & 6 \\
Acinetobacter sp. & & 2 & 2 \\
Staphylococcus aureus & - & 6 & 6 \\
\hline
\end{tabular}

" $n$ " denotes number of patients who required the specified treatment.

(60\%) followed by Klebsiella and Escherichia coli. In patients with late-onset VAP, most common organism isolated was Staphylococcus aureus (26\%), followed closely by $P$. aeroginosa, Klebsiella, E. coli, and Acinetobacter. The overall mortality rates were highest with Pseudomonas (42\%), in spite of adequate and proper antimicrobial therapy, followed by $S$. aureus (33\%) and others.

Mortality was observed to be low in patients who were suffering from potentially remediable conditions, for example, tropical infections, organophosphorus poisoning, Guillain-Barre syndrome, etc. However, a much poorer prognosis was found to be associated with conditions such as sepsis/shock (67\%), pancreatitis (100\%), and conditions with CNS complications (e.g., hydrocephalus [75\%], cerebral malaria [50\%], or meningoencephalitis [50\%]) [Table 7].

Table 6 shows the clinical outcome and its relation with the factors related to patient and their course of care, e.g., age, presence of comorbid factors, infection or immunosuppression, reintubation, tracheostomy, and APACHEIII scores on admission. Nineteen patients out of fifty-one (37\%) succumbed while being treated in CCU. Patients who died were slightly older in age (mean 38.7 years) as against the survivors (mean 31.1 years). Five patients were more than 60 years old and none of them could survive. Thus, mortality was significantly higher in older age groups. Mortality was also higher in the VAP group (Table 8), though the difference was not statisti-

\begin{tabular}{lccc}
\hline Table 6: Mortality and factors related to course of care \\
\hline & $\begin{array}{c}\text { Survivors } \\
\text { (32 patients) }\end{array}$ & $\begin{array}{c}\text { Nonsurvivors } \\
(\mathbf{1 9} \text { patients) }\end{array}$ & $\boldsymbol{P}$ \\
Mean age (years) & 31.1 & 38.7 & \\
Comorbid factors $(n=13)$ & 4 & 9 & $<0.01$ \\
Immunosuppression/ & 4 & 3 & \\
infection $(n=7)$ & & & \\
APACHEIII (SD) & $40.2(22)$ & $65(18.4)$ & $<0.001$ \\
Dialysis/PEEP $(n=11)$ & 4 & 7 & $<0.05$ \\
Reintubation $(n=24)$ & 14 & 10 & \\
Tracheostomy $(n=11)$ & 10 & 1 & $<0.005$ \\
\hline
\end{tabular}

Table 7: Distribution pattern of diseases and mortality

\begin{tabular}{|c|c|c|c|}
\hline Diseases & $\begin{array}{c}n \text { (no. of } \\
\text { patients) }\end{array}$ & Nonsurvivors & Survivors \\
\hline \multicolumn{4}{|l|}{ CNS disorders } \\
\hline Meningoencephalitis & 8 & $4(50 \%)$ & 4 \\
\hline GBS/AIDP & 8 & $1(12 \%)$ & 7 \\
\hline Myasthenia gravis & 1 & - & 1 \\
\hline Hydrocephalus/SAH & 4 & $3(75 \%)$ & 1 \\
\hline \multicolumn{4}{|l|}{ Tropical diseases } \\
\hline Complicated malaria & 2 & 1 & 1 \\
\hline Leptospirosis & 1 & - & 1 \\
\hline Tetanus & 3 & - & 3 \\
\hline \multicolumn{4}{|l|}{ Poisoning } \\
\hline OP poisoning & 11 & $2(18 \%)$ & 9 \\
\hline Snake bite & 1 & 1 & - \\
\hline \multicolumn{4}{|l|}{ Metabolic } \\
\hline ARF/CRF/RTA & 10 & $4(40 \%)$ & 6 \\
\hline DM/DKA & 2 & - & 2 \\
\hline \multicolumn{4}{|l|}{ Miscellaneous } \\
\hline Shock/sepsis & 3 & $2(67 \%)$ & 1 \\
\hline Pancreatitis & 2 & $2(100 \%)$ & - \\
\hline Pulmonary fibrosis & 1 & 1 & - \\
\hline
\end{tabular}

Data expressed as number of patients associated with given factors unless specified.

Total $(n)$ is more than 51 as some patients had multiple disorders. Percentage in parenthesis denotes percentage of patients with the specified disease, who did not survive.

Table 8: VAP and clinical outcome

\begin{tabular}{lccc}
\hline & Total $^{a}$ & Survivors & Nonsurvivors \\
VAP & 24 & 15 & $9(37.5 \%)$ \\
Non-VAP & 23 & 17 & $6^{a}(26 \%)$ \\
& $n=47$ & 32 & 15 \\
\hline
\end{tabular}

${ }^{a}$ Four deaths attributed to ARDS were excluded, and so $n=47$.

cally significant. The attributable risk percentage of VAP was $30.4 \%$ and the relative risk was 1.44 .

This analysis (Table 6) demonstrated that mortality was significantly high in patients with comorbid illnesses $(P<0.01)$ such as hypertension, chronic renal failure, metastatic malignancies, obesity, etc. Mortality was found to be predominantly related to underlying severity of disease which was assessed by APACHEIII scores within first $24 \mathrm{~h}$ of admission to CCU. Mean APACHEIII score on admission in survivor's group was 40.2, whereas it was significantly higher at 65 in nonsurvivors $(P<0.001)$ (Table 6$)$. Significantly $(P<0.005)$ higher survival rates were found in cases that had early and planned tracheostomy, as compared with those who needed reintubations.

\section{Discussion}

VAP is the commonest nosocomial infection amongst patients receiving $\mathrm{MV}$ in $\mathrm{CCU}$. The incidence of VAP in our setting was $47 \%$ and the incidence rate of VAP in our study was 26 per 1000 ventilator days. 
Table 9 shows the incidence of VAP, reported by recent studies (Torres, Kollef, and Fagon), ranging from $15.5 \%$ by Kollef et al. to $27.5 \%$ by Fagon et al. However, it may be noted that both these studies were of 2 and 5 years duration, respectively, with much larger number of patients studied, viz., 277 by Kollef and 1118 by Fagon. The total number of cases in our study is small.

The higher incidence of VAP in our study could also be owing to the presence of comorbid conditions. The patients who succumbed were seriously ill with conditions such as septic shock, pancreatitis, cerebral malaria, etc. The health-seeking behavior of our patients is different compared with that in developed world. Owing to limited resources, patients seek medical help only when it is absolutely inevitable. By the time he is referred to the tertiary-care centre, his underlying condition is well advanced and may be irreversible. This may necessitate longer duration of MV, which is directly proportional to development of VAP.

The other most important factor leading to higher incidence of VAP in our set-up may be attributed to the paucity of nursing staff that leaves lacunae in the ideal patient care. The patient to nurse ratio in our CCU set-up is $4: 1$, the desired ratio being 1:1. Reintubation has resulted in a very high incidence of VAP. An early and planned tracheostomy has shown significant reduction in occurrence of VAP, which is also advocated by many other reports in literature. Other risk factors identified were immunosuppression, use of chemical paralysis, and dialysis. This study also signifies that decline in $\mathrm{PaO}_{2} /$ $\mathrm{FiO}_{2}$ ratio is an early indicator of onset of VAP.

In summary, the significant risk factors for development of VAP were prolonged duration of $\mathrm{MV}$, higher APACHEIII scores on admission signifying severe illness and reintubation. Other positive risk factors identified were presence of comorbid conditions causing immunosuppression/ infection, use of chemical paralysis, and use of dialysis during the course of CCU stay.

The patients who acquired VAP had longer lengths of

CCU stay as well as greater hospital mortality rates (37\%). In our study, VAP was not independently associated with mortality, though mortality rate was higher in patients with VAP (Table 8). Mortality was predominantly associated with underlying severity of disease, presence of comorbid factors, and older age groups. APACHEIII scores within first $24 \mathrm{~h}$ of admission to $\mathrm{CCU}$ were used to address the underlying severity of illness. Patients who developed VAP had statistically higher APACHEIII score at admission (55) as compared with patients without VAP (42). Likewise, patients who died had even higher scores (65), $(P<0.001)$. Thus, APACHEIII score at the time of admission was found to be a useful parameter to prognosticate patients on arrival and also during their course of stay.

Table 10 demonstrates that our CCU has particularly high incidence of pneumonia caused by Pseudomonas and Klebsiella as compared with other studies. Also, we found that incidence of polymicrobial flora was higher in tracheal aspirate culture. Furthermore, mortality rates were higher with Pseudomonas followed by Staphylococcus and Klebsiella. Also, it was observed that pathogens isolated were potentially antibiotic resistant. We could not study Susceptibility profile in detail owing to paucity of clinical microbiology support and unaffording

Table 10: Organisms responsible for VAP (a comparison with other studies) ${ }^{[9]}$

\begin{tabular}{|c|c|c|c|}
\hline & $\begin{array}{c}\text { Fagon et al., } \\
1984\end{array}$ & $\begin{array}{c}\text { Torres et al., } \\
1990\end{array}$ & Our study \\
\hline $\begin{array}{l}\text { No. of episodes of } \\
\text { pneumonia }\end{array}$ & 52 & 25 & 24 \\
\hline $\begin{array}{l}\text { Technique } \\
\text { aspirate }\end{array}$ & PSB & PSB, BAL & Tracheal \\
\hline Threshold (CFU/ml) & $10^{[3]}$ & $10^{[3]}$ & $10^{[6]}$ \\
\hline Gram-negative bacteria & No. (\%) & No. (\%) & No. (\%) \\
\hline$P$. aeroginosa & $16(31)$ & 7 (28) & $11(46)$ \\
\hline Acinetobacter & $8(15)$ & $6(24)$ & $2(8)$ \\
\hline Proteus & $8(15)$ & - & $3(13)$ \\
\hline E. coli & $4(8)$ & $3(12)$ & $3(12)$ \\
\hline Klebsiella & $2(4)$ & $3(12)$ & $7(29)$ \\
\hline \multicolumn{4}{|l|}{ Gram-positive bacteria } \\
\hline S. aureus & $17(33)$ & $5(20)$ & $6(25)$ \\
\hline S. pneum. & $3(6)$ & $1(4)$ & - \\
\hline Polymicrobial flora & $21(40)$ & $10(40)$ & $13(54)$ \\
\hline
\end{tabular}

Table 9: Incidence and mortality rate of VAP ${ }^{[9]}$ (a comparison with other studies)

\begin{tabular}{|c|c|c|c|c|c|}
\hline Authors & Study years & No. of patients studied & Incidence of VAP (\%) & Diagnostic criteria & Mortality rates (\%) \\
\hline Kerver et al. & 1986 & 39 & 67 & Clinical & 30 \\
\hline Torres et al. & 1987-88 & 322 & 24 & Clinical, PSB & 33 \\
\hline Kollef et al. & 1992-93 & 277 & 15.5 & Clinical & 37.5 \\
\hline Fagon et al. & 1989-94 & 1118 & 27.5 & PSB, BAL & 53 \\
\hline Our study & 2003-2004 & 51 & 47 & Clinical & 37 \\
\hline
\end{tabular}


patients but, recounting our experience, our findings are as follows. Pseudomonas was isolated in 11 samples and was found to be maximally susceptible to meropenem and cefpirome. Pseudomonas showed favorable susceptibility pattern to piperacillin, cefoperazone-sulbactam, and amikacin whereas it was fairly resistant to drugs such as levofloxacin, ciprofloxacin, and ceftazidime. K. pneumoniae was isolated in seven samples and showed maximal susceptibility to meropenem, cefpirome, and cefoperazonesulbactam. Klebsiella was moderately susceptible to piperacillin and ciprofloxacin and was highly resistant to amoxicillin-clavulanate, cefotaxime, ceftriaxone, and ceftazidime. S. aureus was isolated in six samples and all isolates were resistant to methicillin and amoxicillinclavulanate. Staphylococcus showed excellent sensitivity to vancomycin and teicoplanin. Linezolid was used only in one patient who did not respond well to vancomycin.

\section{Conclusions}

We arrive at the following conclusions:

1. The incidence of VAP is directly proportional to the duration of MV.

2. Comorbid conditions and reintubation contribute to development of VAP.

3. APACHEIII score on admission is a useful parameter for prognostication of patients.

4. A decline in $\mathrm{PaO}_{2} / \mathrm{FiO}_{2}$ ratio can help in early suspicion of VAP.
5. Early and planned tracheostomy is associated with lower incidence of VAP and low mortality rates.

6. Gram-negative microbes followed by methicillin resistant Staphylococcus aureus are commonest incriminating organisms.

\section{References}

1. Pingleton SK, Fagon JY, Leeper KV. Patient selection for Clinical investigation of VAP. Chest 1992;102;553-8.

2. George DL. Epidemiology of nosocomial pneumonia in MICU. Med Clin Chest Med 1995;16:29-44.

3. Kollef MH, Silver P, Murphy DM. The effect of VAP in determining mortality. Chest 1995;108:1655-62.

4. Sa Borges M, Correa H. Variations in etiology of VAP. Respirator Crit Care Med 1999;160:608-13.

5. Fagon JY, Chastre J, Domart Y, Trouillet JL, Pierre J, Darne C, et al. Nosocomial Pneumonia in patients receiving continuous mechanical ventilation. Am Rev Respirator Dis 1989;139:87784.

6. Fagon J, Chastre J. Evaluation of clinical judgement in identification and treatment of nosocomial pneumonia in ventilated patients. Chest 1993;103:547-53.

7. Pugin J, Auckenthaler R, Mili N. Diagnosis of VAP. Am Rev Respirator Dis 1991;143:1121-9.

8. Knaus WA, Wagner DP, Draper EA, Zimmerman JE, Bergner M. The APACHEIII prognostic system: Risk prediction of hospital mortality for critically ill hospitalized adults. Chest 1991:100:1619-36.

9. Chastre J, Fagon JY. Ventilator-associated pneumonia, Principles of Critical Care; 1998. pp. 617-47. 